\title{
POLYNOMIAL ASYMPTOTIC STABILITY OF DAMPED STOCHASTIC DIFFERENTIAL EQUATIONS
}

\author{
JOHN A. D. APPLEBY AND DANA MACKEY
}

\begin{abstract}
The paper studies the polynomial convergence of solutions of a scalar nonlinear Itô stochastic differential equation

$$
d X(t)=-f(X(t)) d t+\sigma(t) d B(t)
$$

where it is known, a priori, that $\lim _{t \rightarrow \infty} X(t)=0$, a.s. The intensity of the stochastic perturbation $\sigma$ is a deterministic, continuous and square integrable function, which tends to zero more quickly than a polynomially decaying function. The function $f$ obeys $\lim _{x \rightarrow 0} \operatorname{sgn}(x) f(x) /|x|^{\beta}=a$, for some $\beta>1$, and $a>0$. We study two asymptotic regimes: when $\sigma$ tends to zero sufficiently quickly the polynomial decay rate of solutions is the same as for the deterministic equation (when $\sigma \equiv 0$ ). When $\sigma$ decays more slowly, a weaker almost sure polynomial upper bound on the decay rate of solutions is established. Results which establish the necessity for $\sigma$ to decay polynomially in order to guarantee the almost sure polynomial decay of solutions are also proven.
\end{abstract}

\section{INTRODUCTION}

Many authors have contributed to the study of nonexponential rates of decay to equilibrium of solutions stochastic differential equations. The polynomial stability in particular has been the subject of much study, in Mao [7, 8], in Liu and Mao [5, 6] and in Liu [4].

In these works, the authors principally concentrate upon establishing upper bounds on the almost sure rate of convergence of solutions. The equations considered are, in general, nonautonomous equations which are quasilinear in the state variable. The categories of equation studied

Date: 30 September 2003.

1991 Mathematics Subject Classification. Primary: 60H10 Secondary: 34D05, 34F05, 60J60, 93E15.

Key words and phrases. Polynomial asymptotic stability, almost sure asymptotic stability, simulated annealing, diffusion process.

This paper is in final form and no version of it will be submitted for publication elsewhere. 
include those in which there are strong time-dependence in the drift, or in which the diffusion coefficient decays polynomially in time.

As is well known from the theory of ordinary differential equations, slower-than-exponential rates of decay to equilibria can also arise if the restoring force close to the equilibrium is weak (viz., there is no leading order linear term at the equilibrium). This phenomenon has been examined in the stochastic case also, by e.g., Zhang and Tsoi $[12,13]$. In their work, examples are given of stochastic differential equations which converge to equilibrium at a polynomial rate by virtue of the nonlinear form of the drift and diffusion coefficients close to the equilibrium. Other interesting papers in which almost surely globally asymptotically stable solutions of stochastic delay differential equations are found are $[11,10]$, in which the equations studied have general (including polynomial) nonlinearities.

In this work, we attempt to determine the exact almost sure rate of decay for a class of scalar diffusion equations where the drift term is purely state-dependent, and the intensity of the stochastic perturbation is deterministic. To obtain polynomial stability in this class does not require that the perturbation be polynomial: merely that it decays more quickly than some polynomial function. Therefore, polynomial asymptotic stability can arise even in the presence of, for example, a noise perturbation which diminishes exponentially quickly. Thus, the principal mechanism responsible for the slow convergence of solutions is the nonlinear form of the drift term close to the equilibrium.

More precisely, we show that exact polynomial rates of decay can be recovered in the case where the nonlinearity in the drift is responsible for the polynomial convergence of solutions, and when the intensity of the diffusion term decays to zero sufficiently quickly. In cases where the noise term decays more slowly, it is still possible to establish a polynomial rate of decay of solutions, but the bound on rate of decay is related to the rate of decay of the stochastic perturbation only. Finally, we establish a type of converse result: roughly, we show that if the solutions of the stochastic differential equation are polynomially asymptotically stable almost surely, the noise perturbation must decay more quickly than some polynomial function.

We prove these results by expressing the solution of the stochastic differential equation as the sum of a random function independent of 
the solution, and the solution of a perturbed random differential equation whose solution is continuously differentiable. Since the rate of decay of the perturbation can be shown to be the same for almost all paths, the asymptotic behaviour of this random differential equation can be determined by studying the rate of decay of a perturbed deterministic equation. Consequently, a significant part of the paper is devoted to proving results on the decay rate of solutions of deterministic equations. We believe these results may be of independent interest: moreover, we are unaware of the existence elsewhere in the literature of results of the form required here.

A physical motivation for studying this work comes from the problem of simulated annealing. Work on the almost sure stability of diffusion processes modelling annealing has been done, for example, by Chan [2], and Chan and Williams [3]. In these papers, necessary and sufficient conditions for the global almost sure stability of a class of scalar and multidimensional stochastic differential equations were established. The class of equations studied in this paper is included in the works mentioned above by these authors. Some literature concerning the annealing problem is referred to in [2] also.

In future work, we hope to study the rates of decay of solutions of general nonlinear stochastic equations, and also to apply these methods to study the asymptotic decay properties of solutions stochastic functional differential equations with fading external stochastic perturbations. Moreover, as the annealing theory holds in the finite dimensional case, we would expect to be able to extend our analysis to study finite dimensional equations.

\section{Preliminaries}

We first establish some standard notation. As usual, let $x \vee y$ denote the maximum of $x, y \in \mathbb{R}$ and $x \wedge y$ the minimum. The signum function will be denoted by $\operatorname{sgn}$, where $\operatorname{sgn}(x)=1$ for $x>0, \operatorname{sgn}(x)=-1$ for $x<0$ and $\operatorname{sgn}(x)=0$ for $x=0$.

Denote by $C(I ; J)$ be the space of continuous functions from $I$ to $J$, and $C^{1}(I ; J)$ be the corresponding space of all functions with continuous derivatives. Denote by $L\left(\mathbb{R}^{+}\right)$the space of all measurable real-valued functions which are integrable on $\mathbb{R}^{+}$and by $L^{2}\left(\mathbb{R}^{+}\right)$all square integrable functions on $\mathbb{R}^{+}$. 
Let $f \in C(\mathbb{R} ; \mathbb{R})$ and $\sigma \in C\left(\mathbb{R}^{+} ; \mathbb{R}\right) \cap L^{2}\left(\mathbb{R}^{+}\right)$. Furthermore, let $f$ be locally Lipschitz continuous.

Let $\xi \in \mathbb{R}$. Let $\left(\Omega, \mathcal{F},\left(\mathcal{F}^{B}(t)\right)_{t \geq 0}, \mathbb{P}\right)$ be a complete filtered probability space, and $B=\left\{B(t) ; \mathcal{F}^{B}(t) ; 0 \leq t<\infty\right\}$ be a one-dimensional standard Brownian motion on it. The filtration $\left(\mathcal{F}^{B}(t)\right)_{t \geq 0}$ is the natural filtration for standard Brownian motion, viz., $\mathcal{F}^{B}(t)=\sigma\{B(s)$ : $0 \leq s \leq t\}$.

Under these hypotheses, there exists a continuous adapted process $X$ which is a strong solution, up to an explosion time $T_{e}>0$, of the Itô stochastic differential equation

$$
d X(t)=-f(X(t)) d t+\sigma(t) d B(t)
$$

relative to $B$, with initial condition $\xi$, viz. $X$ obeys

$$
\begin{aligned}
& \text { (2.2a) } X(t)=\xi-\int_{0}^{t} f(X(s)) d s+\int_{0}^{t} \sigma(s) d B(s), \quad 0 \leq t<T_{e}, \\
& (2.2 \mathrm{~b}) \quad X(0)=\xi
\end{aligned}
$$

Here, as is conventional, the explosion time $T_{e}$ is defined by

$$
T_{e}=\lim _{n \rightarrow \infty} T_{n}
$$

where $T_{n}=\inf \{t>0:|X(t)|=n\}$.

In order to ensure that $T_{e}(\omega)=\infty$ for almost all sample paths $\omega \in \Omega$ and that almost all solutions converge to zero as $t \rightarrow \infty$ (viz.,

$$
\lim _{t \rightarrow \infty} X(t, \omega)=0, \quad \omega \in \Omega_{0},
$$

where $\mathbb{P}\left[\Omega_{0}\right]=1$ ), the following hypotheses on $f$ and $\sigma$ were imposed in Chan and Williams [3].

Theorem 2.1. Let $f$ be a locally Lipschitz continuous function with

$$
\begin{aligned}
& f \text { strictly increasing on } \mathbb{R}, \\
& \lim _{x \rightarrow \infty} f(x)=\infty, \quad \lim _{x \rightarrow-\infty} f(x)=-\infty \\
& f(0)=0,
\end{aligned}
$$

and suppose $\sigma$ is a continuous function such that

$$
\sigma \text { is decreasing on }[0, \infty), \sigma(0) \text { is finite and } \sigma(t) \rightarrow 0 \text { as } t \rightarrow \infty \text {. }
$$


Then, there is a unique strong solution of $(2.1)$ on $[0, \infty)$, almost surely. If, moreover,

$$
\lim _{t \rightarrow \infty} \sigma(t)^{2} \log (t)=0
$$

then (2.3) also holds.

In the following, we will merely assume that

There is a unique strong solution of $(2.2)$ on $[0, \infty)$

which obeys (2.3)

noting all the time that the hypotheses (2.4), (2.5) suffice to ensure (2.6).

Our interest here is to establish necessary and sufficient conditions for all solutions of (2.1) to converge to zero at a polynomial rate. This notion of almost sure polynomial stability was introduced by Mao in [7] for solutions of nonautonomous nonlinear stochastic differential equations.

Definition 2.2. The process $X$ is almost surely polynomially stable, if there exists a deterministic $\alpha>0$, such that

$$
\limsup _{t \rightarrow \infty} \frac{\log |X(t)|}{\log t} \leq-\alpha, \quad \text { a.s. }
$$

To establish this polynomial stability we will need to impose a decay condition on the fading intensity of the stochastic perturbation $\sigma$, as well as a condition on the behaviour of $f$ close to zero. Before we do this, we establish the first main result of this paper, which does not rely on assumptions of this type.

\section{Structure of Solutions of (2.2)}

In this section we prove that each realisation of the process $X$ can be decomposed into the solution of a perturbed random differential equation (which has its solutions in $C^{1}\left(\mathbb{R}^{+} ; \mathbb{R}\right)$ ) and a random function which is independent of the process $X$. Determining the asymptotic behaviour of almost all realisations of $X$ (in other words, the almost sure asymptotic behaviour of $X$ ) then reduces to studying the asymptotic behaviour of (a) a perturbed ordinary differential equation, and (b) a process whose asymptotic behaviour can be understood by using the law of the iterated logarithm for continuous time martingales. 


\subsection{Representation of solutions of (2.1).}

Theorem 3.1. Suppose $f$ is a locally Lipschitz continuous function which obeys $(2.4 \mathrm{c})$ and

$$
f \in C^{1}(-\delta, \delta) \text { for some } \delta>0 .
$$

Let $\sigma$ be a continuous function with

$$
\sigma \in L^{2}\left(\mathbb{R}^{+}\right)
$$

and suppose that $X$, the solution of (2.1), obeys (2.6). Then, there exists an almost sure set $\Omega^{*} \subseteq \Omega$ such that, for all $\omega \in \Omega^{*}$,

$$
X(t, \omega)=x(t, \omega)+U(t, \omega), \quad t \geq 0,
$$

where

$$
\begin{array}{r}
U(t, \omega)=-\left(\int_{0}^{\infty} \sigma(s) d B(s)-\int_{0}^{t} \sigma(s) d B(s)\right)(\omega) \\
=\left(-\int_{t}^{\infty} \sigma(s) d B(s)\right)(\omega),
\end{array}
$$

and $x(\cdot, \omega)$ is the solution of

$$
x^{\prime}(t, \omega)=-f(x(t, \omega))+g(t, \omega), \quad t \geq 0
$$

which obeys $x(t, \omega) \rightarrow 0$ as $t \rightarrow \infty$, and $g(\cdot, \omega)$ is a continuous function which satisfies

$$
|g(t, \omega)|=\left|f^{\prime}(\eta(t, \omega))\right||U(t, \omega)|
$$

for all $t>T(\omega)$, with $\eta$ obeying

$$
|\eta(t, \omega)-x(t, \omega)| \leq|U(t, \omega)|
$$

In advance of proving this result, we make some comments.

Firstly, the asymptotic behaviour in the case when

$$
f^{\prime}(0)=0
$$

is false is not considered in this work. Results in this direction for the linear equation are well-known and have been studied by many authors. An account of these results on linear equations in the narrow sense is available in e.g., Mao [9].

Secondly, the reformulation of the solution of (2.1) in Theorem 3.1 has certain advantages; if an almost sure estimate on the rate of decay of $U$ can be obtained, the problem reduces to studying the asymptotic behaviour of the function $x$ in (3.4), a problem which, owing to the fact 
that it is defined pathwise, can essentially be studied using the methods of the theory of deterministic ordinary differential equations. However, the study of the asymptotic behaviour of $X$ through $x$ and $U$ must be achieved by studying the asymptotic behaviour of the random functions $x(\cdot, \omega), U(\cdot, \omega)$ for each $\omega$ in an almost sure set. This is because $x(t)$, $U(t)$ are not $\mathcal{F}^{B}(t)$-measurable random variables as $x(t, \omega), U(t, \omega)$ depend on the values of the Brownian motion $B$ on $[t, \infty)$. Hence, $x$, $U$ are not stochastic processes which are adapted to the filtration $\mathcal{F}^{B}$, and so the realisations $t \mapsto X(t, \omega)$ must be studied through the oneparameter families of functions $t \mapsto x(t, \omega), t \mapsto U(t, \omega)$ rather than through "realisations" of $x$ and $U$.

Proof of Theorem 3.1. Introduce the process

$$
Y(t)=\int_{0}^{t} \sigma(s) d B(s)
$$

By (3.1b) and the martingale convergence theorem, there exists an almost sure set $\Omega_{1}$, and a $\mathcal{F}^{B}(\infty)$-measurable random variable $Y^{*}$, such that

$$
\lim _{t \rightarrow \infty} Y(t, \omega)=Y^{*}(\omega)
$$

for all $\omega \in \Omega_{1}$. Define $\int_{0}^{\infty} \sigma(s) d B(s):=Y^{*}$ on $\Omega$. Therefore, for each $\omega \in \Omega_{1}$, the function $t \mapsto U(t, \omega)$ introduced in (3.3) is well-defined. Next, consider the process $Z$ given by $Z(t)=X(t)-Y(t)$ which is well defined for all $\omega \in \Omega_{0}$, the almost sure set in (2.6). Therefore

$$
Z(t)=\xi-\int_{0}^{t} f(X(s)) d s, \quad t \geq 0 .
$$

Since $f$ and $X$ are continuous functions, $t \mapsto Z(t)$ is in $C^{1}((0, \infty) ; \mathbb{R})$ and obeys

$$
Z^{\prime}(t)=-f(Z(t)+Y(t)), \quad t \geq 0 .
$$

Next, let

$$
\Omega_{2}=\left\{\omega \in \Omega_{0}: \lim _{t \rightarrow \infty} X(t, \omega)=0, \lim _{t \rightarrow \infty} Y(t, \omega) \text { exists }\right\} .
$$

By (2.6), (3.1b), this is an almost sure subset of $\Omega_{1}$. Hence, for each $\omega \in \Omega_{2}$ the random function $t \mapsto x(t, \omega)$ given by

$$
x(t, \omega)=Z(t, \omega)+Y^{*}(\omega)=X(t, \omega)+U(t, \omega)
$$

is well-defined. By (3.3), $\lim _{t \rightarrow \infty} U(t, \omega)=0$ for all $\omega \in \Omega_{1}$ and thus $x(t, \omega) \rightarrow 0$ as $t \rightarrow \infty$ for all $\omega \in \Omega_{2}$. Since each path $t \mapsto Z(t, \omega)$ is in 
$C^{1}((0, \infty) ; \mathbb{R})$, the function $t \mapsto x(t, \omega)$ is in $C^{1}((0, \infty) ; \mathbb{R})$ for $\omega \in \Omega_{2}$, and

$$
x^{\prime}(t, \omega)=-f(x(t, \omega)+U(t, \omega)), \quad t \geq 0 .
$$

Define

$$
g(t, \omega)=f(x(t, \omega))-f(x(t, \omega)+U(t, \omega)), \quad t \geq 0 .
$$

Then, as $t \mapsto U(t, \omega)$ is continuous for all $\omega \in \Omega_{2}, t \mapsto g(t, \omega)$ is continuous, and so $t \mapsto x(t, \omega)$ obeys (3.4).

By (3.1a), for each $\omega \in \Omega_{2}$, there exists $T(\omega)>0$ such that, for all $t>T(\omega)$

$$
|x(t, \omega)|<\delta, \quad|x(t, \omega)+U(t, \omega)|<\delta,
$$

because $x(t, \omega) \rightarrow 0, U(t, \omega) \rightarrow 0$ as $t \rightarrow \infty$ for all $\omega \in \Omega_{2}$.

Now, suppose that $U(t, \omega) \geq 0$. Then, by the mean value theorem, for each $t>T(\omega)$, there exists $\eta(t, \omega) \in[x(t, \omega), x(t, \omega)+U(t, \omega)]$ such that

$$
-g(t, \omega)=f(x(t, \omega)+U(t, \omega))-f(x(t, \omega))=f^{\prime}(\eta(t, \omega)) U(t, \omega)
$$

so $|g(t, \omega)|=\left|f^{\prime}(\eta(t, \omega))\right||U(t, \omega)|$. If, on the other hand, $U(t, \omega)<0$, the mean value theorem again implies that for each $t>T(\omega)$, there exists $\eta(t, \omega) \in[x(t, \omega)+U(t, \omega), x(t, \omega)]$ such that

$$
g(t, \omega)=f(x(t, \omega))-f(x(t, \omega)+U(t, \omega))=-f^{\prime}(\eta(t, \omega)) U(t, \omega) .
$$

Thus $|g(t, \omega)|=\left|f^{\prime}(\eta(t, \omega))\right||U(t, \omega)|$. In each case, we have $\mid x(t, \omega)-$ $\eta(t, \omega)|\leq| U(t, \omega) \mid$ as well. Hence (3.5), (3.6) are true, and thus all the claims posited in the statement of the theorem hold.

In the theorem above, it follows that $\eta(t, \omega) \rightarrow 0$ as $t \rightarrow \infty$ for all $\omega$ in an almost sure set. Therefore, as (3.7) holds throughout, it follows that $g(t, \omega)$ tends to zero more quickly than the $X$-independent random function $t \mapsto U(t, \omega)$. Therefore, the size of the perturbation in (3.4) is bounded by the size of $U$. Thus, if a deterministic function $\rho: \mathbb{R}^{+} \rightarrow \mathbb{R}^{+}$can be found so that

$$
\limsup _{t \rightarrow \infty} \frac{|U(t, \omega)|}{\rho(t)}=1
$$

for all $\omega$ in an almost sure set, it follows that

$$
\lim _{t \rightarrow \infty} \frac{g(t, \omega)}{\rho(t)}=0 .
$$


The effect of this is to reduce dramatically the complexity in studying the equation (3.4). In fact, the parameterisation of solutions of (3.4) by $\omega$ becomes redundant when considering asymptotic behaviour, so it is now sufficient to study the asymptotic behaviour of the deterministic ordinary differential equation

$$
x^{\prime}(t)=-f(x(t))+g(t), \quad t \geq 0,
$$

where it is known that $x(t) \rightarrow 0$ as $t \rightarrow \infty$, and the continuous function $g$ decays more quickly to zero than some given function $\rho$. We will turn to the study of such perturbed deterministic ordinary differential equations in the next section.

The question now arises: can such a function $\rho$ be found in (3.9)? This is not only important in helping to determine the asymptotic behaviour of $X$ directly (through the representation of $X$ in formula (3.2)), but also indirectly (through the asymptotic behaviour of the solution of (3.4)). The function $\rho$ required is

$$
\rho(t)=\sqrt{2 \Sigma(t) \log \log \Sigma(t)^{-1}}
$$

where

$$
\Sigma(t)=\int_{t}^{\infty} \sigma(s)^{2} d s
$$

Lemma 3.2. Suppose $\sigma$ is a continuous function obeying

$$
\begin{aligned}
& \Sigma(t)>0 \text { for all } t \geq 0, \\
& \sigma(t)>0 \text { for all } t \geq 0 .
\end{aligned}
$$

Then, with $\rho, \Sigma$ defined in (3.12), (3.13), $U$ defined in (3.3) obeys $(3.9)$.

A more general version of Lemma 3.2, together with a proof, is to be found in [1], also submitted to these Proceedings.

The hypotheses (3.14a) is natural: in the case where (3.14a) does not hold (i.e. $\sigma(t) \equiv 0$ for all $t>T_{0}$ ) the stochastic differential equation (2.1) reduces to the trivial separable differential equation

$$
X^{\prime}(t)=-f(X(t)), \quad t>T_{0}
$$

where only the initial condition $X\left(T_{0}\right)$ is random. On the other hand, the condition (3.14b) (which evidently implies (3.14a)) is a purely technical restriction, which we hope to remove in later work. 


\subsection{Hypotheses on $f$ and $\sigma$; Statement of the Main Results.}

Finally, we mention the hypotheses on $f, \sigma$ used in this paper which deal specifically with the polynomial asymptotic behaviour of solutions of (2.1). We always require $f$ to obey

There exist $\beta>1, a>0$ such that

$$
\lim _{x \rightarrow 0} \frac{f(x) \operatorname{sgn}(x)}{|x|^{\beta}}=a
$$

and $\sigma$ to satisfy the following condition

There exists $\gamma>0$ such that

$$
\gamma=\inf \left\{\alpha>0: \int_{0}^{\infty} s^{2 \alpha} \sigma(s)^{2} d s=\infty\right\}
$$

In (3.16), in the case the set is empty, we define $\gamma=\infty$. This case arises, for example, if $\sigma(t)=\mathrm{e}^{-t}$. We prefer to impose the hypothesis (3.16) rather than a stronger pointwise polynomial bound on $\sigma$, as it is sufficient to establish the a.s. polynomial asymptotic stability of solutions of (2.1). Moreover, as we later prove, the integral condition (3.16) is also necessary if the solution is to be almost surely polynomially stable.

On occasion, we will request that $f$ obeys a stronger restriction than (3.15), namely

There exists $\beta>1, a>0$ such that

$$
\lim _{x \rightarrow 0} \frac{f^{\prime}(x)}{|x|^{\beta-1}}=\beta a \text {. }
$$

Once $x f(x)>0$ for $x \in(-\delta, \delta)$, (3.17) implies (3.15).

The condition (3.15) ensures that $f(x)$ behaves like $x^{\beta}$ as $x \downarrow 0$. However, when $\beta$ is not a rational number, $x^{\beta}$ is not well defined for $x<0$. Therefore, in order to maintain symmetry, we extend $f$ to behave like $-|x|^{\beta}$ as $x \uparrow 0$.

The preservation of symmetry is a crucial hypothesis in the existence of a well-defined decay rate. If the exponent $\beta$ in (3.15) had different values for $x<0$ and $x>0$, the decay rate observed would depend on whether the solution approached zero from above or below. However, in the presence of a stochastic perturbation, it is not clear whether the solution would necessarily be non-oscillatory (that is, whether it ultimately approaches the equilibrium from one side).

We will require a consequence of (3.16) in the next section. 
Lemma 3.3. Let $\beta>1$ and $\gamma>0$ be given by (3.15) and (3.16). If

$$
\gamma>\frac{\beta}{\beta-1}>1
$$

then, for all $\omega \in \Omega^{*}$, an almost sure set, we have

$$
\begin{aligned}
& \lim _{t \rightarrow \infty} t^{\frac{\beta}{\beta-1}} U(t, \omega)=0, \\
& \lim _{t \rightarrow \infty} t^{\frac{\beta}{\beta-1}} g(t, \omega)=0,
\end{aligned}
$$

where $U, g$ are defined by (3.3) and (3.5).

Proof. Since $f \in C^{1}(-\delta, \delta)$ and (3.15) holds, $f^{\prime}(0)=0$. By (3.5) and the fact that $\eta(t, \omega) \rightarrow 0$ as $t \rightarrow \infty$, (3.19a) implies (3.19b). As to (3.19a), note that Lemma 3.2 implies that

$$
\lim _{t \rightarrow \infty} t^{\frac{2 \beta}{\beta-1}} \rho(t)^{2}=0
$$

assures the result. To prove (3.20), first observe that for any $\nu \in$ $(\beta /(\beta-1), \gamma)$

$$
\Sigma(t) \leq \frac{I}{(1+t)^{2 \nu}}, \quad t \geq 0
$$

where $I=\int_{0}^{\infty}(1+s)^{2 \nu} \sigma(s)^{2} d s<\infty$, and $I$ is finite on account of (3.16). Now let $\varepsilon>0$ be any number such that

$$
\frac{\beta}{\beta-1} \frac{1}{\nu}<1-\varepsilon,
$$

where the existence of such an $\varepsilon$ is guaranteed by (3.18). Since $\Sigma(t) \downarrow 0$ as $t \rightarrow \infty$, we have $\Sigma(t)<e^{-e}$ for all $t>T_{1}$. Hence there is

$$
C_{\varepsilon}=\inf _{y \in\left[e^{e}, \infty\right)} \frac{\log \log y}{y^{\varepsilon}}>0
$$

such that, for all $t \geq T_{1}$,

$$
\frac{\rho(t)^{2}}{2}=\Sigma(t) \log \log (\Sigma(t))^{-1} \leq C_{\varepsilon} \Sigma(t)^{1-\varepsilon} .
$$

Now, (3.21) and (3.23) give

$$
\frac{1}{2} t^{\frac{2 \beta}{\beta-1}} \rho(t)^{2} \leq C_{\varepsilon} I^{1-\varepsilon} \frac{1}{(1+t)^{2 \nu(1-\varepsilon)-2 \beta(\beta-1)}},
$$

so (3.22) yields (3.20).

We now state the first main result on asymptotic stability in the paper. 
Theorem 3.4. Suppose that $f$ is a locally Lipschitz continuous function which obeys (2.4c), (3.1a), (3.15), and let $\sigma$ be a positive and continuous function which obeys (3.1b) and (3.16).

If $X$, the strong solution of (2.1) obeys (2.6), and $\beta$ and $\gamma$, the exponents in (3.15) and (3.16), respectively, are related by (3.18), and $a$ is the constant defined in (3.15), then there is a random variable $L$ which assumes either the values 0 or $[a(\beta-1)]^{-1 /(\beta-1)}$, such that

$$
\lim _{t \rightarrow \infty} t^{\frac{1}{\beta-1}}|X(t)|=L, \quad \text { a.s. }
$$

By Theorem 3.1, Lemma 3.3 and the preceding discussion, we see that Theorem 3.4 is a direct consequence of the following result.

Theorem 3.5. Suppose that $f$ is a locally Lipschitz continuous function which obeys (2.4c), (3.1a), (3.15) and $g$ is a continuous function which obeys

$$
\lim _{t \rightarrow \infty} t^{\frac{\beta}{\beta-1}} g(t)=0,
$$

where $\beta>1$ is the exponent in (3.15). Let $x$ be the unique continuous solution of $(3.11)$ on $[0, \infty)$. If

$$
x(t) \rightarrow 0 \text { as } t \rightarrow \infty
$$

and $a$ is the constant defined in (3.15), then there is a constant $L$ which assumes either the values 0 or $[a(\beta-1)]^{-1 /(\beta-1)}$, such that

$$
\lim _{t \rightarrow \infty} t^{\frac{1}{\beta-1}}|x(t)|=L \text {. }
$$

The proof of Theorem 3.5 is the subject of the next section. Before we turn to that proof, let us reflect on the hypotheses of Theorem 3.5 and then Theorem 3.4.

The hypothesis (3.26), which ensures the existence of asymptotically stable solutions of (3.11) when $g(t) \rightarrow 0$ as $t \rightarrow \infty$, is one which can be verified in many cases. To take a concrete example, consider the problem

$$
x^{\prime}(t)=-\operatorname{asgn}(x(t))|x(t)|^{\beta}+g(t), \quad t>0,
$$

where $g(t) \rightarrow 0$ as $t \rightarrow \infty$; we now show that $x(t) \rightarrow 0$ as $t \rightarrow$ $\infty$. Perusal of the explanation below reveals that a nearly identical argument suffices for the problem $x^{\prime}(t)=-f(x(t))+g(t)$, when $f$ is a continuous, odd, and increasing function, with $f(0)=0$.

As can be seen in the proof of Lemma 4.1 below, $x$ obeys

$$
D_{+}|x(t)| \leq-a|x(t)|^{\beta}+|g(t)|, \quad t>0 .
$$


Next, for every $\varepsilon>0$ there is a $T(\varepsilon)>0$ such that $|g(t)|<\varepsilon$ for $t>T(\varepsilon)$. Hence

$$
D_{+}|x(t)| \leq-a|x(t)|^{\beta}+\varepsilon, \quad t>T(\varepsilon) .
$$

If $\bar{x}^{\prime}(t)=-a \bar{x}(t)^{\beta}+\varepsilon, t>T(\varepsilon)$, and $\bar{x}(T(\varepsilon))=1+|x(T(\varepsilon))|$, then $|x(t)| \leq \bar{x}(t)$ for $t \geq T(\varepsilon)$. Thus

$$
\limsup _{t \rightarrow \infty}|x(t)| \leq \lim _{t \rightarrow \infty} \bar{x}(t)=\left(\frac{\varepsilon}{a}\right)^{1 / \beta},
$$

so letting $\varepsilon \downarrow 0$ proves that $x(t) \rightarrow 0$ as $t \rightarrow \infty$.

We now return to discuss Theorem 3.4. When $f$ is a continuous function satisfying (2.4c), (3.15) for some $\beta>1$, all nontrivial asymptotically stable solutions of the deterministic version of equation (2.1) (or, equivalently, the unperturbed version of (3.11)) obey (3.27) with $L=[a(\beta-1)]^{-1 /(\beta-1)}$. Therefore, according to (3.18), when the decay rate of the noise intensity $\sigma$ is sufficiently fast (and so the size of the stochastic perturbation vanishes sufficiently quickly), the asymptotic behaviour of the deterministic and stochastic equations is the same.

We make two comments in relation to this here. First, the result is unsurprising in one respect: if the perturbation vanishes quickly enough we should expect to recover the asymptotic behaviour of the unperturbed problem. However, given that almost all realisations of $X$ are almost everywhere nondifferentiable, it is perhaps surprising that we should recover a $C^{\infty}(1, \infty)$ decay rate $\left(t^{-1 /(\beta-1)}\right)$ for almost all paths. Second, Theorem 3.4 states that the deterministic decay rate is recovered when (3.18) holds i.e., $\beta>\gamma /(\gamma-1)$. Later in this paper, under the hypothesis (3.17), we show that there appears to be a transition from the deterministic asymptotic regime to a new asymptotic regime when $\beta=1 /(\gamma-1)$.

\section{Proof of Theorem 3.5}

We divide the proof of Theorem 3.5 into three steps. Each of these steps will be given in a lemma below. The steps are:

STEP 1: We show that

$$
\limsup _{t \rightarrow \infty} t^{\frac{1}{\beta-1}}|x(t)| \leq\left[\frac{1}{a(\beta-1)}\right]^{\frac{1}{\beta-1}} .
$$


STEP 2: Given STEP 1, we establish that either

$$
\lim _{t \rightarrow \infty} t^{\frac{1}{\beta-1}}|x(t)|=0 \text { or } \limsup _{t \rightarrow \infty} t^{\frac{1}{\beta-1}}|x(t)|=\left[\frac{1}{a(\beta-1)}\right]^{\frac{1}{\beta-1}} .
$$

STEP 3: In the case that

$$
\limsup _{t \rightarrow \infty} t^{\frac{1}{\beta-1}}|x(t)|=\left[\frac{1}{a(\beta-1)}\right]^{\frac{1}{\beta-1}}
$$

we show that

$$
\lim _{t \rightarrow \infty} t^{\frac{1}{\beta-1}}|x(t)|=\left[\frac{1}{a(\beta-1)}\right]^{\frac{1}{\beta-1}} .
$$

Lemma 4.1. (Step 1) Condition (4.1) holds.

Proof. Introduce the function $a: \mathbb{R}^{+} \rightarrow \mathbb{R}$,

$$
a(t)= \begin{cases}\frac{f(x(t))}{|x(t)|^{\beta}}, & x(t)>0, \\ a, & x(t)=0, \\ -\frac{f(x(t))}{|x(t)|^{\beta}}, & x(t)<0 .\end{cases}
$$

Then $a$ is a continuous function which obeys $\lim _{t \rightarrow \infty} a(t)=a$, since $f$ obeys (3.15) and $x$ obeys (3.26). Thus, (3.11) can be written as

$$
x^{\prime}(t)=-a(t) \operatorname{sgn}(x(t))|x(t)|^{\beta}+g(t) .
$$

Indeed, we note that there exists $t_{1}^{*}>0$ such that $a(t)>0$ for all $t>t_{1}^{*}$. Next, note that Theorem 3.5 is trivially true for the case where $x(t)=0$ for all $t$ sufficiently large, so we assume, to the contrary, that $x(t) \not \equiv 0$ on some interval $[T, \infty)$. In this case, we can choose $t^{*}>t_{1}^{*}$ such that $\left|x\left(t^{*}\right)\right|>0$.

Next, we seek a comparison equation for $x$. Fix $t>t^{*}$ and suppose $x(t)>0$. Then, as $x$ is in $C^{1}\left(\mathbb{R}^{+} ; \mathbb{R}\right), x(s)>0$ for all $s \in[t, t+h)$, for $h$ sufficiently small. Hence, by $(4.5)$

$$
\begin{aligned}
|x(t+h)|-|x(t)| & =\int_{t}^{t+h}-a(s)|x(s)|^{\beta} d s+\int_{t}^{t+h} g(s) d s \\
& \leq \int_{t}^{t+h}-a(s)|x(s)|^{\beta} d s+\int_{t}^{t+h}|g(s)| d s .
\end{aligned}
$$

Since $t \mapsto|g(t)|, t \mapsto a(t), t \mapsto|x(t)|^{\beta}$ are continuous, letting $h \downarrow 0$

$$
D_{+}|x(t)| \leq-a(t)|x(t)|^{\beta}+|g(t)|, \quad t \geq t^{*}
$$


wherever $x(t)>0$. In the same manner, for a fixed $t>t^{*}$ where $x(t)<0$, (4.5) yields

$$
\begin{aligned}
|x(t+h)|-|x(t)| & =\int_{t}^{t+h}-a(s)|x(s)|^{\beta} d s-\int_{t}^{t+h} g(s) d s \\
& \leq \int_{t}^{t+h}-a(s)|x(s)|^{\beta} d s+\int_{t}^{t+h}|g(s)| d s
\end{aligned}
$$

so (4.6) holds in the case $x(t)<0$. Finally, if $x(t)=0$, we have

$$
D_{+}|x(t)|=\limsup _{h \rightarrow 0^{+}} \frac{|x(t+h)|-|x(t)|}{h} \leq \limsup _{h \rightarrow 0^{+}}\left|\frac{x(t+h)-x(t)}{h}\right| .
$$

The continuity of the modulus gives

$$
\left|x^{\prime}(t)\right|=\lim _{h \rightarrow 0^{+}}\left|\frac{x(t+h)-x(t)}{h}\right|=\limsup _{h \rightarrow 0^{+}}\left|\frac{x(t+h)-x(t)}{h}\right| .
$$

Thus as $f(x(t))=0$,

$$
D_{+}|x(t)| \leq\left|x^{\prime}(t)\right|=|-f(x(t))+g(t)|=|g(t)|=-a(t)|x(t)|^{\beta}+|g(t)|
$$

and so (4.6) holds when $x(t)=0, t>t^{*}$. Therefore (4.6) holds for all $t \geq t^{*}$.

Next, consider the initial value problem

$$
\begin{aligned}
& \bar{x}^{\prime}(t)=-a(t) \bar{x}(t)^{\beta}+|g(t)|, \quad t>t^{*}, \\
& \bar{x}\left(t^{*}\right)=\left|x\left(t^{*}\right)\right|+1 .
\end{aligned}
$$

(4.7) has a unique continuous solution on $\left(t^{*}, \infty\right)$ and, by the comparison principle, $|x(t)| \leq \bar{x}(t), t \geq t^{*}$. We now obtain a bound on the solution of (4.7) by considering the unperturbed version of (4.7), namely

$$
\begin{aligned}
& \phi^{\prime}(t)=-a(t) \phi(t)^{\beta}, \quad t \geq t^{*}, \\
& \phi\left(t^{*}\right)=\left|x\left(t^{*}\right)\right|+1 .
\end{aligned}
$$

Then $\bar{x}(t) \geq \phi(t), t \geq t^{*}$. The asymptotic behaviour of the solution of (4.8) is easily obtained by quadrature, noting that $a(t) \rightarrow a$ as $t \rightarrow \infty$. Indeed

$$
\lim _{t \rightarrow \infty} t^{\frac{1}{\beta-1}} \phi(t)=\left[\frac{1}{a(\beta-1)}\right]^{\frac{1}{\beta-1}} .
$$

Therefore, as $\bar{x}(t) \geq \phi(t)$, (4.9) and (3.25) imply

$$
0 \leq \limsup _{t \rightarrow \infty} \frac{|g(t)|}{\bar{x}(t)^{\beta}} \leq \limsup _{t \rightarrow \infty} \frac{|g(t)|}{\phi(t)^{\beta}}=\limsup _{t \rightarrow \infty} \frac{t^{\frac{\beta}{\beta-1}}|g(t)|}{\left(t^{\frac{1}{\beta-1}} \phi(t)\right)^{\beta}}=0
$$


so $\lim _{t \rightarrow \infty} g(t) /|\bar{x}(t)|^{\beta}=0$. Hence, (4.7) implies

$$
\lim _{t \rightarrow \infty} \frac{\bar{x}^{\prime}(t)}{\bar{x}(t)^{\beta}}=-a
$$

as $a(t) \rightarrow a$ as $t \rightarrow \infty$. Integration now gives

$$
\lim _{t \rightarrow \infty} t^{\frac{1}{\beta-1}} \bar{x}(t)=\left[\frac{1}{a(\beta-1)}\right]^{\frac{1}{\beta-1}} .
$$

Since $|x(t)| \leq \bar{x}(t)$, we have established (4.1).

Lemma 4.2. (Step 2) (4.2) holds.

Proof. Let $L=(a(\beta-1))^{-1 /(\beta-1)}$. According to (4.1), there exists $0 \leq L_{0} \leq L$ such that

$$
\limsup _{t \rightarrow \infty} t^{\frac{1}{\beta-1}}|x(t)|=L_{0} .
$$

If $L_{0}=0$, we have the first part of (4.2). Suppose now that $L_{0} \in(0, L)$. Then for every $\varepsilon \in\left(0, L-L_{0}\right)$ there is $T_{1}(\varepsilon)>0$ such that

$$
t^{\frac{1}{\beta-1}}|x(t)| \leq L_{0}+\varepsilon, \quad t \geq T_{1}(\varepsilon) .
$$

By (3.25), it follows that $\int_{t}^{\infty}|g(s)| d s$ is well defined for all $t \geq 0$, and moreover, as $t^{\frac{\beta}{\beta-1}}|x(t)|^{\beta} \leq\left(L_{0}+\varepsilon\right)^{\beta}$, we have that $\int_{t}^{\infty} a(s)|x(s)|^{\beta} d s$ is well defined for every $t \geq 0$. By (3.26), (4.5), we have

So

$$
-x(t)=\int_{t}^{\infty}-a(s) \operatorname{sgn}(x(s))|x(s)|^{\beta} d s+\int_{t}^{\infty} g(s) d s,
$$

$$
t^{\frac{1}{\beta-1}}|x(t)| \leq t^{\frac{1}{\beta-1}} \int_{t}^{\infty}|a(s)||x(s)|^{\beta} d s+t^{\frac{1}{\beta-1}} \int_{t}^{\infty}|g(s)| d s .
$$

Next, for every $\varepsilon \in(0, a)$, there is $T_{2}(\varepsilon)>0$ such that $|a(t)|<a+\varepsilon$ for all $t>T_{2}(\varepsilon)$. Now, let $\varepsilon \in\left(0, a \wedge\left(L-L_{0}\right)\right)$ and $T(\varepsilon)=T_{1}(\varepsilon) \vee T_{2}(\varepsilon)$.

Then for $t>T(\varepsilon)$, by (4.11), we have

$$
\begin{aligned}
t^{\frac{1}{\beta-1}}|x(t)| & \leq t^{\frac{1}{\beta-1}} \int_{t}^{\infty} \frac{1}{s^{\frac{\beta}{\beta-1}}} s^{\frac{\beta}{\beta-1}}|x(s)|^{\beta} d s(a+\varepsilon)+t^{\frac{1}{\beta-1}} \int_{t}^{\infty}|g(s)| d s \\
& \leq(a+\varepsilon)\left(L_{0}+\varepsilon\right)^{\beta}(\beta-1)+t^{\frac{1}{\beta-1}} \int_{t}^{\infty} g(s) d s .
\end{aligned}
$$

Therefore,

$$
L_{0}=\limsup _{t \rightarrow \infty} t^{\frac{1}{\beta-1}}|x(t)| \leq(a+\varepsilon)\left(L_{0}+\varepsilon\right)^{\beta}(\beta-1) .
$$

Letting $\varepsilon \downarrow 0$ yields $L_{0} \leq a L_{0}^{\beta}(\beta-1)$, so $L_{0} \geq L$. But this contradicts $L_{0}<L$. Thus in (4.10), either $L_{0}=0$ or $L_{0}=L$, as needed in (4.2) 
Lemma 4.3. (Step 3) Condition (4.3) holds.

Proof. Fix $C \in(0, L)$ and choose $\varepsilon>0$ sufficiently small, so that

$$
0<\varepsilon<\frac{\frac{C}{\beta-1}-a C^{\beta}}{C^{\beta}+2^{\frac{\beta}{\beta-1}}} \vee 1 \text {. }
$$

This implies

$$
-\frac{C}{\beta-1}+a C^{\beta}+\varepsilon\left(C^{\beta}+2^{\frac{\beta}{\beta-1}}\right)<0 .
$$

Since $\lim _{t \rightarrow \infty} a(t)=a$ and $\lim _{t \rightarrow \infty} t^{\beta /(\beta-1)}|g(t)|=0$, it follows that there exists $T_{0}(\varepsilon)>0$ such that $a(t)<a+\varepsilon$ and $t^{\beta /(\beta-1)}|g(t)|<\varepsilon$ for all $t>T_{0}(\varepsilon)$. Also, since $\lim \sup _{t \rightarrow \infty} t^{1 /(\beta-1)}|x(t)|=L$, there exists $T_{2}(\varepsilon)>0$ and a sequence $\left(t_{n}\right)_{n \geq 1}$ with $\lim _{n \rightarrow \infty} t_{n}=\infty$ such that

$$
t_{n}^{\frac{1}{\beta-1}}\left|x\left(t_{n}\right)\right|>C(1+\varepsilon)^{-\frac{1}{\beta-1}}
$$

for all $t_{n}>T_{2}(\varepsilon)$. This made possible by the fact that $L>C(1+\varepsilon)^{-\frac{1}{\beta-1}}$.

Now, choose $T(\varepsilon)$ to be the smallest member of this sequence which is greater than $T_{0}(\varepsilon)$ so $T(\varepsilon)>T_{0}(\varepsilon)$ and

$$
T(\varepsilon)^{\frac{1}{\beta-1}}|x(T(\varepsilon))|>C(1+\varepsilon)^{-\frac{1}{\beta-1}} .
$$

Let $T_{1}(\varepsilon)=\varepsilon T(\varepsilon)$ and define

$$
x_{L}(t)=C\left(t+T_{1}(\varepsilon)\right)^{-\frac{1}{\beta-1}}
$$

for all $t \geq T(\varepsilon)$.

Note that $|x(T(\varepsilon))|>0$. Consider first the case when $x(T(\varepsilon))>0$. In this case (4.14) and (4.13) imply that $x(T(\varepsilon))>x_{L}(T(\varepsilon))$.

Then, as $T(\varepsilon)>T_{0}(\varepsilon)$, for $t>T(\varepsilon)$ we have

$$
\begin{aligned}
& x_{L}^{\prime}(t)+a(t) \operatorname{sgn}\left(x_{L}(t)\right) x_{L}(t)^{\beta}+|g(t)| \\
& =\left(t+T_{1}(\varepsilon)\right)^{-\frac{\beta}{\beta-1}}\left[-\frac{C}{\beta-1}+a(t) C^{\beta}+\left(t+T_{1}(\varepsilon)\right)^{\frac{\beta}{\beta-1}}|g(t)|\right] \\
& \quad<\left(t+T_{1}(\varepsilon)\right)^{-\frac{\beta}{\beta-1}}\left[-\frac{C}{\beta-1}+(a+\varepsilon) C^{\beta}+\varepsilon\left(\frac{T(\varepsilon)+T_{1}(\varepsilon)}{T(\varepsilon)}\right)^{\frac{\beta}{\beta-1}}\right] \\
& \quad<\left(t+T_{1}(\varepsilon)\right)^{-\frac{\beta}{\beta-1}}\left[-\frac{C}{\beta-1}+(a+\varepsilon) C^{\beta}+\varepsilon(1+\varepsilon)^{\frac{\beta}{\beta-1}}\right] \\
& \quad<\left(t+T_{1}(\varepsilon)\right)^{-\frac{\beta}{\beta-1}}\left[-\frac{C}{\beta-1}+(a+\varepsilon) C^{\beta}+\varepsilon 2^{\frac{\beta}{\beta-1}}\right]<0,
\end{aligned}
$$


since $\varepsilon \in(0,1)$ and $\varepsilon$ obeys (4.12). Therefore, if $x(T(\varepsilon))>0,|x(t)|=$ $x(t)>x_{L}(t)$ for all $t>T(\varepsilon)$.

Now suppose that $x(T(\varepsilon))<0$. Then, letting $z(t)=-x(t)$, we get

$$
z^{\prime}(t)=-a(t) \operatorname{sgn}(z(t))|z(t)|^{\beta}-g(t), \quad t>T(\varepsilon) .
$$

Note by (4.13) that we have

$$
T(\varepsilon)^{\frac{1}{\beta-1}} z(T(\varepsilon))>C(1+\varepsilon)^{-\frac{1}{\beta-1}} .
$$

Defining $z_{L}(t)=x_{L}(t)$ for $t \geq T(\varepsilon)$, we see that $z(T(\varepsilon))>z_{L}(T(\varepsilon))$ and

$$
z_{L}^{\prime}(t)<-a(t) \operatorname{sgn}\left(z_{L}(t)\right) z_{L}(t)^{\beta}-|g(t)|,
$$

for all $t>T(\varepsilon)$. Therefore, we have

$$
0<z_{L}(t)<z(t)=-x(t)=|x(t)| .
$$

Hence $|x(t)|>x_{L}(t), t \geq T(\varepsilon)$.

Therefore, in both cases we have $|x(t)|>x_{L}(t)$ for all $t \geq T(\varepsilon)$ and it follows easily that

$$
\liminf _{t \rightarrow \infty} t^{\frac{1}{\beta-1}}|x(t)| \geq C
$$

Letting $C \uparrow L$ yields $\liminf _{t \rightarrow \infty} t^{\frac{1}{\beta-1}}|x(t)| \geq L$ and hence

$$
\lim _{t \rightarrow \infty} t^{\frac{1}{\beta-1}}|x(t)|=L
$$

by the second part of (4.2).

Remark 4.4. In the theorem above, both the cases where $L=0$ and $L=$ $(a(\beta-1))^{-1 /(\beta-1)}$ can be realised, even when the order of magnitude of the perturbation remains the same as $t \rightarrow \infty$. Indeed, the initial value problem

$$
\begin{aligned}
& x_{1}^{\prime}(t)=-\operatorname{sgn}\left(x_{1}(t)\right) x_{1}(t)^{2}-\frac{t+\frac{1}{\sqrt{2}}-\frac{1}{4}}{\left(t+\frac{1}{\sqrt{2}}\right)^{4}}, \quad t>0, \\
& x_{1}(0)=1
\end{aligned}
$$

obeys all the hypotheses of the theorem and has the unique solution

$$
x_{1}(t)=\frac{1}{2}\left(t+\frac{1}{\sqrt{2}}\right)^{-2},
$$

for all $t \geq 0$. Clearly, this solution satisfies

$$
\lim _{t \rightarrow \infty} t x_{1}(t)=0,
$$

so, for this problem, $L=0$. 
On the other hand, the unique solution of the initial value problem

$$
\begin{aligned}
& y^{\prime}(t)=-y(t)^{2}-\frac{1}{(1+t)^{3}}, \quad t>0, \\
& y(0)=1
\end{aligned}
$$

can be expressed in terms of Bessel functions and can easily be shown to satisfy

$$
\lim _{t \rightarrow \infty} t y(t)=1
$$

Moreover, it can be shown that $y(t)>0$ for all $t>0$. Hence this solution also satisfies the initial value problem

$$
\begin{aligned}
& x_{2}^{\prime}(t)=-x_{2}(t)^{2} \operatorname{sgn}\left(x_{2}(t)\right)-\frac{1}{(1+t)^{3}}, \quad t>0, \\
& x_{2}(0)=1 .
\end{aligned}
$$

We have thus obtained an example where $L=(a(\beta-1))^{-1 /(\beta-1)}$, as for this problem $a=1$ and $\beta=2$. Note that in both examples the initial condition is the same and the perturbation has the same decay rate, i.e.

$$
\lim _{t \rightarrow \infty} t^{3} g(t)=-1
$$

Remark 4.5. It is always possible to get an arbitrarily fast rate of decay for the solution of

$$
\begin{aligned}
& x^{\prime}(t)=-f(x(t))+g(t), \quad t>0 \\
& x(0)=x_{0},
\end{aligned}
$$

provided that the perturbation has the appropriate form and rate of decay. Indeed, a rate of decay $d(t)$ can be obtained, where $d \in C^{1}([0, \infty))$ and obeys $d(0)=1$ and $\frac{d(t)}{d^{\prime}(t)} \rightarrow 0$ as $t \rightarrow \infty$. The last condition implies that $d$ decays to zero faster than any exponential function. If the perturbation is

$$
g(t)=x_{0} d^{\prime}(t)+f\left(x_{0} d(t)\right)
$$

then $\lim _{t \rightarrow \infty} \frac{g(t)}{-d^{\prime}(t)}=x_{0}$ and the solution of the initial value problem is $x(t)=x_{0} d(t)$.

\section{Asymptotic Behaviour of (2.1) With Slowly Decaying \\ NOISE}

We now consider the asymptotic behaviour of (2.1) when the intensity of the stochastic perturbation fades more slowly. First, we note that the perturbation $U$ decays at a polynomial rate of at least $-\gamma$. 
Lemma 5.1. Let $\beta>1$ and $\gamma>0$ be given by (3.16). If

$$
\gamma \leq \frac{\beta}{\beta-1}
$$

then $U$ defined by (3.3) obeys

$$
\limsup _{t \rightarrow \infty} \frac{\log |U(t, \omega)|}{\log t} \leq-\gamma
$$

for all $\omega \in \Omega^{*}$, an almost sure set.

Proof. Let $0<\nu<\gamma$, and note that the estimates (3.21), (3.23) still hold. Let $T_{1}, C_{\varepsilon}>0$ be as defined in Lemma 3.3, where $\varepsilon>0$ is sufficiently small. Hence for $t>T_{1}$,

$$
\rho(t)^{2} \leq 2 C_{\varepsilon} \Sigma(t)^{1-\varepsilon} \leq 2 C_{\varepsilon} \frac{I^{1-\varepsilon}}{(1+t)^{2 \nu(1-\varepsilon)}}
$$

Therefore

$$
\limsup _{t \rightarrow \infty} \frac{\log \rho(t)}{\log t} \leq-\nu(1-\varepsilon) .
$$

Letting $\varepsilon \downarrow 0$, and then $\nu \uparrow \gamma$ gives

$$
\limsup _{t \rightarrow \infty} \frac{\log \rho(t)}{\log t} \leq-\gamma
$$

Finally, as (3.9) holds for a.a. $\omega$ in an almost sure set,

$$
\limsup _{t \rightarrow \infty} \frac{\log |U(t, \omega)|}{\log t}=\limsup _{t \rightarrow \infty} \frac{\log \rho(t)}{\log t} \leq-\gamma
$$

thus proving (5.2).

The next result establishes the rate of decay of equation (3.11) when the perturbation $g$ is bounded by a slowly decaying polynomial.

Lemma 5.2. Suppose $f$ is a locally Lipschitz continuous function which obeys (2.4c), (3.15). Let $\alpha \leq \beta /(\beta-1)$, where $\beta>1$ is the exponent in (3.15), and let $g$ be a continuous function which obeys

$$
\limsup _{t \rightarrow \infty} \frac{\log |g(t)|}{\log t} \leq-\alpha
$$

Let $x$ be the unique continuous solution of $(3.11)$ on $[0, \infty)$. If $x$ obeys (3.26), then

$$
\limsup _{t \rightarrow \infty} \frac{\log |x(t)|}{\log t} \leq-\frac{\alpha}{\beta}
$$


Proof. Equation (3.11) can be written as

$$
x^{\prime}(t)=-a(t) \operatorname{sgn}(x(t))|x(t)|^{\beta}+g(t), \quad t \geq 0
$$

where $a$ is continuous and $\lim _{t \rightarrow \infty} a(t)=a$. Thus $a(t)>a / 2$ for $t>T_{1}^{\prime \prime}$. Next, for all $\varepsilon \in(0, \alpha)$ there is $T_{1}^{\prime}(\varepsilon)>0$ such that $t>T_{1}^{\prime}(\varepsilon)$ implies $|g(t)|<t^{-\alpha+\frac{\varepsilon}{2}}$. Now, let $T_{2}^{\prime}(\varepsilon)=\inf \left\{t>T_{1}(\varepsilon) \vee T_{1}^{\prime \prime} \vee 1: x(t) \neq 0\right\}$. If the set is empty, the result is proven, as the result is trivially true in the case $x(t)=0$ for all $t>T_{1} \vee T_{1}^{\prime \prime} \vee 1$. Suppose the set is not empty. Then there exists $T_{2}(\varepsilon)>T_{2}^{\prime}(\varepsilon)$ such that $\left|x\left(T_{2}(\varepsilon)\right)\right|>0$. Let $x_{\varepsilon}=x\left(T_{2}(\varepsilon)\right)$. If $\bar{x}\left(T_{2}(\varepsilon)\right)=x_{\varepsilon}$ and

$$
\bar{x}^{\prime}(t)=-a(t)|\bar{x}(t)|^{\beta}+|g(t)|, \quad t \geq T_{2}(\varepsilon),
$$

then $|x(t)| \leq \bar{x}(t)$ for $t \geq T_{2}(\varepsilon)$, where, of course, the function $\bar{x}$ is uniquely determined.

Since $\alpha \leq \beta /(\beta-1)$, for every $\varepsilon>0$, we have $\alpha-\varepsilon<\beta /(\beta-1)$, so $0>\alpha-\varepsilon-\frac{\alpha-\varepsilon}{\beta}-1$. Since $\beta>1$, there exists $M>1$ such that

$$
\frac{a}{2} M^{\beta} x_{\varepsilon}^{\beta} T_{2}(\varepsilon)^{\alpha-\varepsilon}-\frac{\alpha-\varepsilon}{\beta} M x_{\varepsilon} T_{2}(\varepsilon)^{\frac{\alpha-\varepsilon}{\beta}-1}>1
$$

Finally, we define

$$
x_{u}(t)=M x_{\varepsilon}\left(\frac{t}{T_{2}(\varepsilon)}\right)^{-\frac{\alpha-\varepsilon}{\beta}}, \quad t \geq T_{2}(\varepsilon)
$$

so that $x_{u}\left(T_{2}(\varepsilon)\right)=M x_{\varepsilon}$. Since $M>1, x_{u}\left(T_{2}(\varepsilon)\right)>x\left(T_{2}(\varepsilon)\right)$. For $t>T_{2}(\varepsilon)>1,(5.5)$ implies

$$
t^{\varepsilon / 2}\left[\frac{a}{2} M^{\beta} x_{\varepsilon}^{\beta} T_{2}(\varepsilon)^{\alpha-\varepsilon}-\frac{\alpha-\varepsilon}{\beta} M x_{\varepsilon} T_{2}(\varepsilon)^{\frac{\alpha-\varepsilon}{\beta}} t^{\alpha-\varepsilon-\frac{\alpha-\varepsilon}{\beta}-1}\right]>1 .
$$

Thus, for $t>T_{2}(\varepsilon)$,

$$
\frac{a}{2} M^{\beta} x_{\varepsilon}^{\beta} T_{2}(\varepsilon)^{\alpha-\varepsilon} t^{-\alpha+\varepsilon}-\frac{\alpha-\varepsilon}{\beta} M x_{\varepsilon} T_{2}(\varepsilon)^{\frac{\alpha-\varepsilon}{\beta}} t^{-\frac{\alpha-\varepsilon}{\beta}-1}>t^{-\alpha+\frac{\varepsilon}{2}},
$$


so giving the estimate

$$
\begin{aligned}
x_{u}^{\prime}(t)+a(t) \operatorname{sgn}\left(x_{u}(t)\right)\left|x_{u}(t)\right|^{\beta}= & M x_{\varepsilon} T_{2}(\varepsilon)^{\frac{\alpha-\varepsilon}{\beta}} t^{-\frac{\alpha-\varepsilon}{\beta}-1} \cdot \frac{-(\alpha-\varepsilon)}{\beta} \\
& +a(t) M^{\beta} x_{\varepsilon}^{\beta}\left(\frac{t}{T_{2}(\varepsilon)}\right)^{-(\alpha-\varepsilon)} \\
> & \frac{a}{2} M^{\beta} x_{\varepsilon}^{\beta} T_{2}(\varepsilon)^{\alpha-\varepsilon} t^{-\alpha+\varepsilon} \\
& -\frac{\alpha-\varepsilon}{\beta} M x_{\varepsilon} T_{2}(\varepsilon)^{\frac{\alpha-\varepsilon}{\beta}} t^{-\frac{\alpha-\varepsilon}{\beta}-1} \\
& >t^{-\alpha+\frac{\varepsilon}{2}}>|g(t)| .
\end{aligned}
$$

Therefore, $x_{u}$ obeys the differential inequality

$$
\begin{aligned}
x_{u}^{\prime}(t) & >-a(t) \operatorname{sgn}\left(x_{u}(t)\right)\left|x_{u}(t)\right|^{\beta}+|g(t)|, \quad t \geq T_{2}(\varepsilon), \\
x_{u}\left(T_{2}(\varepsilon)\right) & >\bar{x}\left(T_{2}(\varepsilon)\right)>0 .
\end{aligned}
$$

Since $x_{u}\left(T_{2}(\varepsilon)\right)>\bar{x}\left(T_{2}(\varepsilon)\right)>0$, either $x_{u}(t)>\bar{x}(t)$ for all $t \geq T_{2}(\varepsilon)$, or as $\bar{x}$ and $x_{u}$ are $C^{1}$, there exists $T^{*}>T_{2}(\varepsilon)$ such that $x_{u}(t)>\bar{x}(t)$ for $T_{2}(\varepsilon) \leq t<T^{*}, 0<x_{u}\left(T^{*}\right)=\bar{x}\left(T^{*}\right)$. Suppose such a finite $T^{*}$ exists. Then $\bar{x}^{\prime}\left(T^{*}\right) \geq x_{u}^{\prime}\left(T^{*}\right)$, so we have

$$
\begin{aligned}
\bar{x}^{\prime}\left(T^{*}\right) & =-a\left(T^{*}\right) \operatorname{sgn}\left(x\left(T^{*}\right)\right)\left|x\left(T^{*}\right)\right|^{\beta}+\left|g\left(T^{*}\right)\right| \\
& =-a\left(T^{*}\right) \operatorname{sgn}\left(x_{u}\left(T^{*}\right)\right)\left|x_{u}\left(T^{*}\right)\right|^{\beta}+\left|g\left(T^{*}\right)\right| .
\end{aligned}
$$

Therefore

$$
x_{u}^{\prime}\left(T^{*}\right) \leq \bar{x}^{\prime}\left(T^{*}\right)=-a\left(T^{*}\right) \operatorname{sgn}\left(x_{u}\left(T^{*}\right)\right)\left|x_{u}\left(T^{*}\right)\right|^{\beta}+\left|g\left(T^{*}\right)\right|<x_{u}^{\prime}\left(T^{*}\right),
$$

which is a contradiction. Therefore $x_{u}(t)>\bar{x}(t)$ for all $t>T_{2}(\varepsilon)$. Hence

$$
|x(t)| \leq x_{u}(t)=M x_{\varepsilon}\left(\frac{t}{T_{2}(\varepsilon)}\right)^{-\frac{(\alpha-\varepsilon)}{\beta}}, \quad t \geq T_{2}(\varepsilon) .
$$

Hence

$$
\limsup _{t \rightarrow \infty} \frac{\log |x(t)|}{\log t} \leq-\frac{\alpha-\varepsilon}{\beta} .
$$

Letting $\varepsilon \downarrow 0$ now gives the result.

Lemmas 5.1 and 5.2 enable us to prove first a result on the decay rate of solutions of (2.1).

Theorem 5.3. Suppose that $f$ is a locally Lipschitz continuous function which obeys (2.4c), (3.1a) and (3.15). Let $\sigma$ be a positive and continuous function which obeys (3.1b) and (3.16). 
If $X$, the strong solution of (2.1) obeys (2.6) and $\gamma$ and $\beta$, the exponents in (3.15) and (3.16), respectively, are related by (5.1), then

$$
\limsup _{t \rightarrow \infty} \frac{\log |X(t)|}{\log t} \leq-\frac{\gamma}{\beta}, \quad \text { a.s. }
$$

Proof. According to Lemma 5.1, $U(\cdot, \omega)$ obeys (5.2). By (3.5), and (5.2) the function $g(\cdot, \omega)$ obeys

$$
\limsup _{t \rightarrow \infty} \frac{\log |g(t, \omega)|}{\log t} \leq-\gamma
$$

By Lemma 5.2 and Theorem 3.1, the function $x(\cdot, \omega)$ defined by $(3.2)$, and which obeys (3.4), obeys

$$
\limsup _{t \rightarrow \infty} \frac{\log |x(t, \omega)|}{\log t} \leq-\frac{\gamma}{\beta}
$$

Hence, by (5.8) and (5.9), (3.2) implies

$$
\limsup _{t \rightarrow \infty} \frac{\log |X(t, \omega)|}{\log t} \leq-\frac{\gamma}{\beta},
$$

since $\beta>1$. In all the above $\omega$ is in an almost sure set, so (5.7) holds.

Taking the results of Theorems 3.1 and 5.3 together suggests that the solution of $(2.1)$ decays at a polynomial rate of at least $-1 /(\beta-1)$ for $\gamma \in\left(\frac{\beta}{\beta-1}, \infty\right)$ and at a rate of at least $-\gamma / \beta$ for $\gamma \in\left(0, \frac{\beta}{\beta-1}\right]$. However, with a slightly stronger hypothesis on $f$, it is possible to show that the decay rate of $-1 /(\beta-1)$ can be extended to the interval $\gamma \in\left(\frac{1}{\beta-1}, \infty\right)$ and the rate on the interval $\gamma \in\left(0, \frac{1}{\beta-1}\right]$ can be improved from $-\gamma / \beta$ to $-\gamma$.

To obtain these refined estimates on the decay rate requires a different approach, and we outline this method first before proving the results.

The main thrust of this idea is to apply Lemmas 5.1 and 5.2 successively to the equation (3.4), each time improving the estimate on the polynomial rate of decay of solutions of (3.4). The improvement is possible because the perturbation $g$ in (3.4) is of the form

$$
|g(t)|=\left|f^{\prime}(\eta(t))\right||U(t)|
$$

and, due to (3.6), $|\eta(t)| \leq|x(t)|+|U(t)|$. Thus an a priori estimate on the rate of decay of $\eta$ is known. Therefore as the polynomial behaviour of the function $f^{\prime}$ is known close to zero (as prescribed in (3.17)), a bound on the decay rate of $f^{\prime}(\eta(t))$ can be estimated, and so a more 
rapid rate of decay of $g$ can be established. Due to Lemma 5.2, this ensures that a faster rate of decay of $x$ can be estimated, and this in turn, enables a faster rate of decay of $g$ to be established. Continuing in this manner we can determine the optimal rate of decay of the solution of (3.4), and hence that of (2.1).

Lemma 5.4. Let $f$ be locally Lipschitz continuous and obey $(2.4 \mathrm{c})$, (3.1a), (3.15) and (3.17). Let $\sigma$ be a positive and continuous function which obeys (3.1b) and (3.16). Let $\omega \in \Omega^{*}$, an almost sure set and $x(\cdot, \omega)$ be the function defined by (3.2) which obeys $x(t, \omega) \rightarrow 0$ as $t \rightarrow \infty$. If $\beta$ and $\gamma$, the exponents in (3.15) and (3.16), respectively, satisfy the inequality

$$
\frac{1}{\beta-1}<\gamma \leq \frac{\beta}{\beta-1},
$$

then the following hold:

(i)

$$
\limsup _{t \rightarrow \infty} \frac{\log |x(t, \omega)|}{\log t} \leq-\frac{\gamma}{\beta}
$$

(ii) Suppose there is $c_{0} \geq \gamma / \beta$ such that

$$
\limsup _{t \rightarrow \infty} \frac{\log |x(t, \omega)|}{\log t} \leq-c_{0}
$$

Then one of the following holds:

(a) If $c_{0}>\frac{1}{\beta-1}\left(\frac{\beta}{\beta-1}-\gamma\right)$, then

$$
\lim _{t \rightarrow \infty} t^{\frac{1}{\beta-1}}|x(t, \omega)|=L(\omega)
$$

where $L(\omega)$ is either 0 or $[a(\beta-1)]^{-1 /(\beta-1)}$.

(b) If $c_{0} \leq \frac{1}{\beta-1}\left(\frac{\beta}{\beta-1}-\gamma\right)$, then

$$
\limsup _{t \rightarrow \infty} \frac{\log |x(t, \omega)|}{\log t} \leq-\frac{(\beta-1) c_{0}+\gamma}{\beta} .
$$

Proof. Part (i) (or (5.11)) holds by (5.9) in Theorem 5.3. To prove part (ii), suppose (5.12) holds, where $c_{0} \geq \gamma / \beta$. Then (3.6) implies

$$
|\eta(t, \omega)| \leq|\eta(t, \omega)-x(t, \omega)|+|x(t, \omega)| \leq|U(t, \omega)|+|x(t, \omega)|,
$$

so it follows that

$$
\limsup _{t \rightarrow \infty} \frac{\log |\eta(t, \omega)|}{\log t} \leq-\left(c_{0} \wedge \gamma\right)
$$


by Lemma 5.1 and equation (5.12). Since $\eta(t, \omega) \rightarrow 0$ as $t \rightarrow \infty$, by (3.17), we have

$$
\begin{aligned}
& \limsup _{t \rightarrow \infty} \frac{\log \mid f^{\prime}(\eta(t, \omega) \mid}{\log t} \\
& =\limsup _{t \rightarrow \infty}\left[\frac{\left.\log \left|f^{\prime}(\eta(t, \omega)) /\right| \eta(t, \omega)\right|^{\beta-1} \mid}{\log t}+(\beta-1) \frac{\log |\eta(t, \omega)|}{\log t}\right] \\
& =(\beta-1) \limsup _{t \rightarrow \infty} \frac{\log |\eta(t, \omega)|}{\log t} \leq-(\beta-1)\left(c_{0} \wedge \gamma\right) .
\end{aligned}
$$

Therefore, according to Lemma 5.1 and (3.5),

$$
\limsup _{t \rightarrow \infty} \frac{\log |g(t, \omega)|}{\log t} \leq-\left\{(\beta-1) c_{0} \wedge \gamma+\gamma\right\} \text {. }
$$

If $\underline{c_{0} \geq \gamma}, c_{0} \wedge \gamma=\gamma$ so (5.10) and (5.15) imply

$$
\limsup _{t \rightarrow \infty} \frac{\log |g(t, \omega)|}{\log t} \leq-\beta \gamma<-\frac{\beta}{\beta-1} .
$$

Hence $(3.25)$ holds for $g(\cdot, \omega)$ and we may apply Theorem 3.5 to $x(\cdot, \omega)$ (which is the solution of (3.4)) to conclude (5.13).

If $\gamma>c_{0}>\frac{1}{\beta-1}\left(\frac{\beta}{\beta-1}-\gamma\right)$ (which is possible as (5.10) is true), then

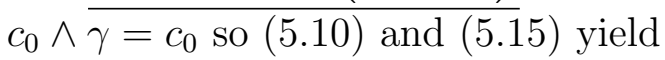

$$
\limsup _{t \rightarrow \infty} \frac{\log |g(t, \omega)|}{\log t} \leq-\left((\beta-1) c_{0}+\gamma\right)<\frac{-\beta}{\beta-1} .
$$

As in the case above, (5.13) is true. Hence we have established alternative (a).

If $c_{0} \leq \frac{1}{\beta-1}\left(\frac{\beta}{\beta-1}-\gamma\right),(5.10)$ implies $c_{0}<\gamma$ so $c_{0} \wedge \gamma=c_{0}$ and we have

$$
\limsup _{t \rightarrow \infty} \frac{\log |g(t, \omega)|}{\log t} \leq-\left((\beta-1) c_{0}+\gamma\right)=:-\alpha
$$

Hence $\alpha \leq \beta /(\beta-1)$. We may now apply Lemma 5.2 to $x(\cdot, \omega)$, the solution of (3.4), to give (5.14).

Lemma 5.4 deals with the case when the noise perturbation fades reasonably quickly. The next result achieves the corresponding effect when the decay rate of the noise perturbation is slower.

Lemma 5.5. Let $f$ be locally Lipschitz continuous and obey (2.4c), (3.1a), (3.15), (3.17). Let $\sigma$ be a positive and continuous function which obeys (3.1b) and (3.16). Let $\omega \in \Omega^{*}$, an almost sure set and $x(\cdot, \omega)$ be the function defined by (3.2) which obeys $x(t, \omega) \rightarrow 0$ as $t \rightarrow \infty$. If 
$\beta$ and $\gamma$, the exponents in (3.15) and (3.16), respectively, satisfy the inequality

$$
\gamma \leq \frac{1}{\beta-1}
$$

then the following hold

(i) (5.11) is true.

(ii) Suppose there is $c_{0} \geq \gamma / \beta$ such that (5.12) holds. Then one of the following is true:

(a) If $c_{0} \geq \gamma$ then

$$
\limsup _{t \rightarrow \infty} \frac{\log |x(t, \omega)|}{\log t} \leq-\gamma .
$$

(b) If $c_{0}<\gamma$, then (5.14) holds.

Proof. The proof of Part (i) is the same as that of Lemma 5.4, part (i). To prove Part (ii), note first that the estimate (5.15) still holds for $g$.

Let $\underline{c_{0} \geq \gamma}$. then $c_{0} \wedge \gamma=\gamma$, so by (5.15),

$$
\limsup _{t \rightarrow \infty} \frac{\log |g(t, \omega)|}{\log t} \leq-\beta \gamma=:-\alpha
$$

Then (5.16) implies $\alpha \leq \beta /(\beta-1)$. We may now apply Lemma 5.2 to $x(\cdot, \omega)$, the solution of $(3.4)$, to give (5.17). This establishes part (a). As to part (b), suppose $\underline{c_{0}<\gamma}$. Then $c_{0} \wedge \gamma=c_{0}$, so (5.15) implies

$$
\limsup _{t \rightarrow \infty} \frac{\log |g(t, \omega)|}{\log t} \leq-\left((\beta-1) c_{0}+\gamma\right)=:-\alpha^{\prime} .
$$

Then, as $c_{0}<\gamma$ and (5.16) holds, $\alpha^{\prime}<\beta \gamma$, so $\alpha^{\prime}<\beta /(\beta-1)$. We may now apply Lemma 5.2 to $x(\cdot, \omega)$, the solution of $(3.4)$, to give $(5.14)$. This establishes part (b) of the result.

We now apply Lemmas 5.3, 5.4 iteratively to prove the following result.

Lemma 5.6. Let $f$ be locally Lipschitz continuous and obey $(2.4 \mathrm{c})$, (3.1a), (3.15), (3.17). Let $\sigma$ be a positive and continuous function which obeys (3.1b) and (3.16). Let $\omega \in \Omega^{*}$, an almost sure set and $x(\cdot, \omega)$ be the function defined by (3.2) which obeys $x(t, \omega) \rightarrow 0$ as $t \rightarrow \infty$.

(i) If $\beta$ and $\gamma$ (the exponents in (3.15) and (3.16), respectively) are related by (5.10), then (5.13) holds.

(ii) If $\beta$ and $\gamma$ are related by (5.16), then (5.17) holds. 
Proof. Define the sequence $\left(c_{n}\right)_{n \geq 0}$ as follows: let $c_{0}=\frac{\gamma}{\beta}, c_{n+1}=$ $\frac{\beta-1}{\beta} c_{n}+\frac{\gamma}{\beta}, n \geq 0$. Then

$$
\gamma-c_{n+1}=\frac{\beta-1}{\beta}\left(\gamma-c_{n}\right), \quad n \geq 0
$$

so $c_{n} \uparrow \gamma$ as $n \rightarrow \infty$.

Consider case (i), where $\frac{1}{\beta-1}<\gamma \leq \frac{\beta}{\beta-1}$. By Lemma 5.4(ii), we can then see that if

$$
\limsup _{t \rightarrow \infty} \frac{\log |x(t, \omega)|}{\log t} \leq-c_{n}
$$

then

$$
c_{n}>\frac{1}{\beta-1}\left(\frac{\beta}{\beta-1}-\gamma\right)
$$

implies (5.13) and

$$
c_{n} \leq \frac{1}{\beta-1}\left(\frac{\beta}{\beta-1}-\gamma\right)
$$

implies

$$
\limsup _{t \rightarrow \infty} \frac{\log |x(t, \omega)|}{\log t} \leq-c_{n+1} .
$$

Note however, that because $c_{n} \uparrow \gamma$ as $n \rightarrow \infty$, for every $\varepsilon>0$ there is an $N_{\varepsilon} \in \mathbb{N}$ such that $c_{N_{\varepsilon}}>\gamma-\varepsilon$. Now, as $\gamma>(\beta-1)^{-1}$, there exists $\varepsilon>0$ such that $\gamma>\frac{1}{\beta-1}+\varepsilon \frac{\beta-1}{\beta}$. Thus

$$
c_{N_{\varepsilon}}>\gamma-\varepsilon>\frac{1}{\beta-1}\left(\frac{\beta}{\beta-1}-\gamma\right) .
$$

Hence (5.13) holds and Part (i) has been established.

Consider now Part (ii), where $\gamma \leq \frac{1}{\beta-1}$. By Lemma 5.5(ii), since $c_{n}<\gamma$ for all $n \in \mathbb{N}$, we see that, if

$$
\limsup _{t \rightarrow \infty} \frac{\log |x(t, \omega)|}{\log t} \leq-c_{n}
$$

then

Therefore, for all $n \in \mathbb{N}$,

$$
\limsup _{t \rightarrow \infty} \frac{\log |x(t, \omega)|}{\log t} \leq-c_{n+1}
$$

$$
\limsup _{t \rightarrow \infty} \frac{\log |x(t, \omega)|}{\log t} \leq-c_{n}
$$

Letting $n \rightarrow \infty$, as $c_{n} \uparrow \gamma$, we have (5.17) and Part (ii) has been established.

We now have all the elements to prove a result when (5.1) holds, under the stronger assumption that $f$ obeys (3.17). 
Theorem 5.7. Suppose that $f$ is a locally Lipschitz continuous function and obeys (2.4c), (3.1a), (3.15), (3.17). Let $\sigma$ be a positive and continuous function which obeys (3.1b) and (3.16). Let $X$ be the strong solution of (2.1) which obeys (2.6).

(i) If $\beta$ and $\gamma$, the exponents in (3.15) and (3.16), respectively, are related by (5.10) then there is a random variable $L$, which assumes either of the values $L=0$ or $L=[a(\beta-1)]^{-1 /(\beta-1)}$, such that

$$
\lim _{t \rightarrow \infty} t^{\frac{1}{\beta-1}}|X(t)|=L, \quad \text { a.s. }
$$

(ii) If $\beta$ and $\gamma$ are related by (5.16) then

$$
\limsup _{t \rightarrow \infty} \frac{\log |X(t)|}{\log t} \leq-\gamma, \quad \text { a.s. }
$$

Proof. To prove Part (i), note by Lemma 5.6 that the function $x(\cdot, \omega)$ obeys (5.13) for all $\omega$ in an almost sure set, and Lemma 5.1 implies that

$$
\limsup _{t \rightarrow \infty} \frac{\log |U(t, \omega)|}{\log t} \leq-\gamma<-\frac{1}{\beta-1},
$$

since (5.10) holds. Therefore

$$
\lim _{t \rightarrow \infty} t^{\frac{1}{\beta-1}}|U(t, \omega)|=0 .
$$

By (5.13), (5.20), (3.2), for all $\omega$ in an almost sure set we have

$\limsup _{t \rightarrow \infty} t^{\frac{1}{\beta-1}}|X(t, \omega)| \leq \limsup _{t \rightarrow \infty}\left(t^{\frac{1}{\beta-1}}|x(t, \omega)|+t^{\frac{1}{\beta-1}}|U(t, \omega)|\right)=L(\omega)$, and

$$
\liminf _{t \rightarrow \infty} t^{\frac{1}{\beta-1}}|X(t, \omega)| \geq \liminf _{t \rightarrow \infty}\left(t^{\frac{1}{\beta-1}}|x(t, \omega)|-t^{\frac{1}{\beta-1}}|U(t, \omega)|\right)=L(\omega),
$$

where $L$ is the random variable from Lemma 5.4. Hence, (5.18) holds.

As to the proof of Part (ii), using Lemma 5.6, we see that the function $x(\cdot, \omega)$ obeys $(5.17)$ for all $\omega$ in an almost sure set. Hence (3.2) and Lemma 5.1 imply that

$$
\limsup _{t \rightarrow \infty} \frac{\log |X(t, \omega)|}{\log t} \leq-\gamma
$$

for all $\omega$ in an almost sure set, which is (5.19).

It is interesting to note that the polynomial decay rate of solutions is $-\frac{1}{\beta-1} \wedge \gamma$; therefore the decay rates as $\gamma$ approaches $\frac{1}{\beta-1}$ from above, and when $\gamma$ approaches $\frac{1}{\beta-1}$ from below, are equal, so the estimates on the decay rates match as the problem passes from the "small noise" 
parameter region to the "large noise" parameter region. It appears, moreover, that $\gamma=1 /(\beta-1)$ is the critical case. In view of the improved estimates available through Theorem 5.7 it is reasonable to ask whether the decay rate in the "large noise" region could again be improved, and whether the size of this parameter region could be further reduced. Although we cannot prove that these results are optimal, numerical simulations appear to confirm, for the problem with $f(x)=a \operatorname{sgn}(x)|x|^{\beta}$ and $\sigma(t)=(1+t)^{-\left(\frac{1}{2}+\gamma\right)}(\beta>1, a>0, \gamma>0)$, that we have

$$
\limsup _{t \rightarrow \infty} \frac{\log |X(t)|}{\log t} \leq-\gamma, \quad \text { a.s. }
$$

for $\gamma \in\left(0, \frac{1}{\beta-1}\right]$, while for $\gamma \in\left(\frac{1}{\beta-1}, \infty\right)$,

$$
\lim _{t \rightarrow \infty} t^{\frac{1}{\beta-1}}|X(t)|=\left[\frac{1}{a(\beta-1)}\right]^{\frac{1}{\beta-1}}, \quad \text { a.s. }
$$

\section{Necessity of Condition (3.16) For Polynomial Stability}

By Theorem 3.1 and Theorem 5.3 we have shown that it is sufficient for $\sigma$ to obey condition (3.16) in order for solutions of (2.1) to be almost surely polynomially stable. We now show that a condition of the form (3.16) is also necessary to ensure almost sure polynomial stability.

Theorem 6.1. Suppose that $f$ is a locally Lipschitz continuous function and obeys (2.4c), (3.1a), (3.15). Let $\sigma$ be a positive and continuous function which obeys (3.1b) and suppose $X$ is the strong solution of (2.1) which obeys (2.6).

Consider the statements:

(6.1) There is $\gamma>0$ such that $\gamma=\inf \left\{\alpha>0: \int_{0}^{\infty} t^{2 \alpha} \sigma(t)^{2} d t=\infty\right\}$, and

$$
\text { There is } \alpha>0 \text { such that } \limsup _{t \rightarrow \infty} \frac{\log |X(t)|}{\log t} \leq-\alpha \text {, a.s. }
$$

Then

(i) (6.1) implies (6.2), where $\alpha=\frac{1}{\beta-1} \wedge \frac{\gamma}{\beta}$.

(ii) If $\alpha>\frac{1}{\beta}$, (6.2) implies (6.1), with $\gamma \geq \alpha \wedge(\alpha \beta-1)$.

Proof. Part (i) follows directly from Theorem 3.1 and Theorem 5.3. We concentrate on the proof of (ii). Using integration by parts on (2.1) 
and rearranging, we have

$$
\begin{aligned}
\int_{0}^{t}(1+s)^{\nu} & \sigma(s) d B(s)=(1+t)^{\nu} X(t)-X(0) \\
& -\int_{0}^{t} \nu(1+s)^{\nu-1} X(s) d s+\int_{0}^{t}(1+s)^{\nu} f(X(s)) d s
\end{aligned}
$$

where we choose $\nu \in(0, \alpha \wedge(\alpha \beta-1))$. By $(6.2)$

$$
\limsup _{t \rightarrow \infty} \frac{\log (1+t)^{\nu}|X(t)|}{\log t} \leq \nu-\alpha<0, \quad \text { a.s. }
$$

so the first two terms on the righthand side of (6.3) have a finite limit as $t \rightarrow \infty$, almost surely. Since (6.2) implies

$$
\limsup _{t \rightarrow \infty} \frac{\log (1+t)^{\nu-1}|X(t)|}{\log t} \leq \nu-1-\alpha<-1, \quad \text { a.s. }
$$

it follows that $(1+t)^{\nu-1}|X(t)| \in L^{1}\left(\mathbb{R}^{+}\right)$a.s. Hence the third term on the righthand side of (6.3) has a finite limit as $t \rightarrow \infty$, almost surely. By (3.15), (6.2) and $\nu<\alpha \beta-1$, we have

$$
\begin{aligned}
\limsup _{t \rightarrow \infty} \frac{\log (1+t)^{\nu}|f(X(t))|}{\log (1+t)} \\
\quad=\limsup _{t \rightarrow \infty}\left[\nu+\frac{\log \left[|f(X(t))| /|X(t)|^{\beta}\right]}{\log (1+t)}+\beta \frac{\log |X(t)|}{\log (1+t)}\right] \\
\leq \nu-\alpha \beta<-1, \quad \text { a.s. }
\end{aligned}
$$

Therefore $(1+t)^{\nu}|f(X(t))| \in L^{1}\left(\mathbb{R}^{+}\right)$, a.s. Thus, the fourth term on the righthand side of (6.3) has a finite limit as $t \rightarrow \infty$, almost surely. Since all the terms on the righthand side of (6.3) have finite limits as $t \rightarrow \infty$, almost surely we have that

$$
\lim _{t \rightarrow \infty} \int_{0}^{t}(1+s)^{\nu} \sigma(s) d B(s) \quad \text { exists a.s. }
$$

and the limit is a.s. finite. This in turn implies that

$$
\int_{0}^{\infty}(1+s)^{2 \nu} \sigma(s)^{2} d s<\infty
$$

Thus (6.1) holds with $\gamma>\nu \in(0, \alpha \wedge(\alpha \beta-1))$, or $\gamma \geq \alpha \wedge(\alpha \beta-1)$, as required. 


\section{REFERENCES}

[1] J. A. D. Appleby. Almost sure subexponential decay rates of Itô-Volterra equations. Electron. J. Qual. Th. Differ. Equations., submitted, 2003.

[2] T. Chan. On multidimensional annealing problems. Math. Proc. Camb. Phil. Soc., 105:177-184, 1989.

[3] T. Chan and D. Williams. An 'excursion' approach to an annealing problem. Math. Proc. Camb. Phil. Soc., 105:169-176, 1989.

[4] K. Liu. Some remarks on exponential stability of stochastic differential equations. Stochastic Anal. Appl., 19(1):59-65, 2001.

[5] K. Liu, X. Mao. Exponential stability of non-linear stochastic evolution equations. Stochastic Process. Appl., 78:173-193, 1998.

[6] K. Liu, X. Mao. Large time behaviour of dynamical equations with random perturbation features. Stochastic Anal. Appl., 19(2):295-327, 2001.

[7] X. Mao. Almost sure polynomial stability for a class of stochastic differential equations. Quart. J. Math. Oxford Ser. (2), 43(2):339-348, 1992.

[8] X. Mao. Polynomial stability for perturbed stochastic differential equations with respect to semimartingales. Stochastics Process. Appl., 41:101-116, 1992,

[9] X. Mao. Stochastic Differential Equations and Applications. Horwood Series in Mathematics and Applications. Horwood, Chichester, 1997.

[10] A. Rodkina. On the asymptotic stability of nonlinear stochastic delay equations Cubo Journal, to appear, 2(4), 2004.

[11] A. Rodkina, V. Nosov. On stability of stochastic delay cubic equations. Proc. Dynam. Systems Appl., submitted, 2003.

[12] B. Zhang, A. H. Tsoi. Lyapunov functions in weak exponential stability and controlled stochastic systems. J. Ramanujan Math. Soc., 11(2):85-102, 1996.

[13] B. Zhang, A. H. Tsoi. Weak exponential asymptotic stability of stochastic differential equations. Stochastic Anal. Appl., 15(4):643-649, 1997.

Centre for Modelling with Differential Equations (CMDE), School of Mathematical Sciences, Dublin City University, Dublin 9, Ireland E-mail address: john.appleby@dcu.ie

URL: http://webpages.dcu.ie/ applebyj

Centre for Modelling with Differential Equations (CMDE), School of Mathematical Sciences, Dublin City University, Dublin 9, Ireland

E-mail address: dana.mackey@dcu.ie

$U R L:$ http://webpages.dcu.ie/〜mackeyd 